\title{
A new antihypertensive drug ameliorate insulin resistance
}

Yan-xia LIU

Acta Pharmacologica Sinica (2012) 33: 429-430; doi: 10.1038/aps.2012.31

I nsulin resistance (IR) is defined as decreased sensitivity and/or responsiveness to insulin that promote glucose disposal. A growing body of clinical and epidemiologic evidence indicates that essential hypertension and IR often coexist $^{[1]}$. Approximately 50 percent of patients with hypertension can be considered to have IR and hyperinsuline$\mathrm{mia}^{[1]}$. This inextricable linkage between hypertension and IR has been identified to increase the prevalence of cardiovascular disease (CVD) and new onset of type II diabetes that is the major cause of morbidity and mortality in this clinical syndrome ${ }^{[2]}$. However, the driving force linking IR and hypertension remains to be fully elucidated.

In the current first-line antihypertensive agents, various drugs have been shown to have different glycometabolic effects. Diuretics or beta blockers have been proved to decrease insulin sensitivity and subsequently deteriorate IR states, whereas calcium channel blockers have been investigated to be neutral ${ }^{[3]}$. Only angiotensin-converting enzyme (ACE) inhibitors and angiotensin II (Ang II) type 1 (AT1) receptor antagonists have been reported to enhance insulin sensitivity and reduce new onset of type II diabetes in hypertensive individuals as

Department of Pharmacology, School of Basic Medical Sciences, Tianjin Medical University, Tianjin 300070, China

(liuyanxia@tmu.edu.cn) highlighted in a recent meta-analysis ${ }^{[4]}$. Indeed, there is not even consensus as to whether or not there are pronounced benefits on IR after ARBs treatments. Bahadir et al reported that the effects of telmisartan on IR were neutral in hypertensive patients with the metabolic syndrome $^{[5]}$. Despite intense research, drugs able to ameliorate hypertension and, at the same time, improve IR and/ or hyperinsulinemia are still lacking. It is a great value to develop a new generation of antihypertensive drugs with advantages in improving metabolic disorders.

Endothelial dysfunction, characterized by impaired endothelium-independent vascular relaxation and an imbalance between decreased nitric oxide (NO) release and enhanced ET-1 production, is commonly observed in patients with hypertension, diabetes, hyperlipidemia and other cardiovascular diseases, and has been considered to be an underlying pathophysiological mechanism that served as the link between IR and hypertension ${ }^{[6,7]}$. On one hand, endothelial dysfunction associated with IR states is featured by injured insulin-stimulated $\mathrm{NO}$ release from endothelium with decreased blood flow and reduced delivery of substrates and hormones to insulin target tissues ${ }^{[8]}$. On the other hand, the imbalance between endothelial-derived relaxing and contracting factors is a potential contributor to the abnormal modulation of vascular tone and the pathogenesis of high blood pressure $^{[6]}$. Thus, the pharmacological approach to restoring endothelial function is expected to be a feasible way to ameliorate IR associated with hypertension. Actually, results of the current basic research strongly support that the improvements of IR states in hypertension after ACEIs or ARBs medications are mainly due to their protection against endothelial dysfunction ${ }^{[7]}$.

Endothelial cells express a diverse array of ion channels which play important roles in modulating cell functions. It has been recognized that activation of endothelial ATP-sensitive potassium channels $\left(\mathrm{K}_{\mathrm{ATP}}\right)$ can modulate the progression of endothelial dysfunction. Opening of endothelial $\mathrm{K}_{\mathrm{ATP}}$ channels can regulate intracellular $\mathrm{Ca}^{2+}$ levels that affect the production and release of endothelial autacoids, eg, NO, ET-1, and prostaglandins ${ }^{[6]}$. Theoretically, endothelial $\mathrm{K}_{\text {ATP }}$ channel openers are predicted to be able to inhibit the development of IR in hypertensive patients.

An article by WANG et $a l^{[9]}$ in recent issue of Acta Pharmacologica Sinica demonstrates for the first time that iptakalim, a novel selective $\mathrm{K}_{\text {АTP }}$ channel opener, could prevent the establishment and progression of IR with hypertension in animal models via keeping the balance between NO and ET-1 signaling in endothelial cells. WANG et al show that treating the fructose-fed rats (FFRs) with iptakalim for 4 weeks lowered 
blood pressure and restored the balance between the production of $\mathrm{NO}$ and secretion of ET-1. Furthermore, the impaired ACh-induced endothelium-dependent relaxation of aortic tissue derived from the FFRs was improved significantly after iptakalim administration. Of note, using the euglycemic-hyperinsulinemia clamp analysis, they confirmed that iptakalim can improve insulin sensitive index (ISI), normalize the glucose infusion rate (GIR), and decrease plasma insulin levels in FFRs. In spontaneously hypertensive rats (SHRs), besides effectively lowering blood pressure, they also found that iptakalim treatment ameliorated IR states. These pharmacological characteristics implicates that iptakalim is suitable for the treatment of hypertension associated with IR. Interestingly, iptakalim could significantly increase the release of $\mathrm{NO}$ and expression of eNOS protein and inhibit the production of ET-1 in PI3-kinase inhibitor wortmannin and hyperinsulinemia stimulated human umbilical vein endothelial cells (HUVECs). These in vitro results indicate that iptakalim could protect against IR-induced endothelial dysfunction. Based on these observations, they conclude that activation of $\mathrm{K}_{\mathrm{ATP}}$ channel by iptakalm ameliorate IR associated with hypertension via restoring the balance between NO and ET-1 signaling in endothelial cells.

Abundant previous pharmacological studies have demonstrated that iptakalim is a highly selective $\mathrm{K}_{\text {ATP }}$ channel opener, which can preferentially relax small arteries and effectively reduce blood pressure ${ }^{[10,11]}$. Another article published in Acta Pharmacologica Sinica suggested that iptakalim, via activating $\mathrm{K}_{\mathrm{ATP}}$ channels, enhances the endothelial chemerin/ChemR23 axis and NO production and thus improves endothelial function $^{[12]}$. They also claim that there is a casual relationship between the antihypertensive response of iptakalim and KCNJ11 polymorphisms in Chinese Han hypertensive patients ${ }^{[13]}$. The study around of iptakalim has attracted widespread concern in the field of cardiovascular research $^{[6,14,15]}$.

Notably, Wang et al provided the experimental evidence that iptakalim was able to ameliorate IR states with hypertension though restoring endothelial function in animals ${ }^{[9]}$. Because metabolic disorders such as IR and/ or hyperinsulinemia and type II diabetes are often observed in hypertensive individuals, it is possible that iptakalm may exhibit additional benefits in these patients. Their most interesting finding uncovers the advanced property of iptakalim and is a valuable contribution in the field of hypertension and IR therapy.

1 Reaven GM. Insulin resistance/compensatory hyperinsulinemia, essential hypertension, and cardiovascular disease. J Clin Endocrinol Metab 2003; 88: 2399-403.

2 Sowers JR. Insulin resistance and hypertension. Am J Physiol Heart Circ Physiol 2004; 286: H1597602.

3 Taylor EN, Hu FB, Curhan GC. Antihypertensive medications and the risk of incident type 2 diabetes. Diabetes Care 2006; 29: 1065-70.

4 Garcia-Puig J, Ruilope LM, Luque M, Fernandez J, Ortega R, Dal-Re R. AVANT Study Group Investigators. Glucose metabolism in patients with essential hypertension. Am J Med 2006; 119: 318-26.

5 Bahadir O, Uzunlulu M, Oguz A, Bahadir MA. Effects of telmisartan and losartan on insulin resistance in hypertensive patients with metabolic syndrome. Hypertens Res 2007; 30: 49-53.

6 Minamino T, Hori M. Protecting endothelial function: A novel therapeutic target of ATP-sensitive potassium channel openers. Cardiovasc Res 2007; 73: 448-9.

7 Kim JA, Montagnani M, Koh KK, Quon MJ. Reciprocal relationships between insulin resistance and endothelial dysfunction: molecular and pathophysiological mechanisms. Circulation 2006; 113: 1888-904.

8 Vincent MA, Montagnani M, Quon MJ. Molecular and physiologic actions of insulin related to production of nitric oxide in vascular endothelium. Curr Diab Rep 2003; 3: 279-88.

9 Wang Y, Zeng FH, Long CL, Pan ZY, Cui WY, Wang $\mathrm{RH}$, et al. The novel ATP-sensitive potassium channel opener iptakalim prevents insulin resistance associated with hypertension via restoring endothelial function. Acta Pharmacol Sin 2011; 32: 1466-74.

10 Wang $\mathrm{H}$. Cardiovascular ATP-sensitive potassium channel as a new molecular target for development of antihypertensive drugs. Acta Pharmacol Sin 1998; 19: 397-402.

11 Pan Z, Huang J, Cui W, Long C, Zhang Y, Wang $\mathrm{H}$. Targeting hypertension with a new adenosine triphosphate-sensitive potassium channel opener iptakalim. J Cardiovasc Pharmacol 2010; 56: 215-28.

12 Duan RF, Cui WY, Wang H. Association of the antihypertensive response of iptakalim with KCNJ11 (Kir6.2 gene) polymorphisms in Chinese Han hypertensive patients. Acta Pharmacol Sin 2011; 32: 1078-84.

13 Zhao RJ, Wang H. Chemerin/ChemR23 signaling axis is involved in the endothelial protection by $\mathrm{K}_{\text {ATP }}$ channel opener iptakalim. Acta Pharmacol Sin 2011; 32: 573-80.

14 Costa AD. Iptakalim: a new or just another KCO? Cardiovasc Res 2009; 83: 417-8.

15 Sikka P, Kapoor S, Bindra VK, Saini M, Saxena KK. Iptakalim: A novel multi-utility potassium channel opener. J Pharmacol Pharmacother 2012; 3: 12-4. 